\title{
Archivos de Cardiología de México

\section{Síndrome cardiorrenal tipo 1 en la unidad de cuidados intensivos coronarios del Hospital Nacional Arzobispo Loayza}

\author{
Paul M. Preza ${ }^{\mathrm{a}, *}$, Abdías Hurtado ${ }^{\mathrm{b}}$, Victoria Armas $^{\mathrm{a}}$ y César P. Cárcamo ${ }^{\mathrm{c}}$ \\ a Servicio de cardiología, Hospital Nacional Arzobispo Loayza, Lima, Perú \\ b Servicio de nefrología, Hospital Nacional Arzobispo Loayza, Lima, Perú \\ c Facultad de salud pública y administración, Universidad Peruana Cayetano Heredia, Lima, Perú
}

Recibido el 19 de agosto de 2014; aceptado el 21 de mayo de 2015

\author{
PALABRAS CLAVE \\ Síndrome \\ cardiorrenal; \\ Insuficiencia \\ cardíaca; \\ Insuficiencia renal; \\ Mortalidad \\ hospitalaria; \\ Perú
}

\begin{abstract}
Resumen
Objetivos: Evaluar la incidencia de síndrome cardiorrenal tipo 1 (SCR1) en una unidad de cuidados intensivos coronarios y su asociación a mortalidad intrahospitalaria a 30 días, así como a otras características epidemiológicas.

Métodos: Se revisaron las historias clínicas de todos los pacientes hospitalizados con diagnóstico de falla cardíaca aguda en un periodo de 4 años. Se definió como SCR1 la presencia de falla cardíaca aguda más una creatinina al ingreso $\geq 0.3 \mathrm{mg} / \mathrm{dL}$ con respecto a la creatinina basal calculada por la fórmula MDRD75, y/o una elevación $\geq 50 \%$ de la creatinina al ingreso en un periodo de $48 \mathrm{~h}$.

Resultados: La incidencia de SCR1 fue del 27.87\%, IC95\%: 20.13-36.71 (34 de 122). Hubo una mayor frecuencia de SCR1 en los pacientes que ingresaron con diagnóstico de shock cardiogénico (RR: 2.02; IC95\%: 1.20-3.93; $p=0.0378$ ) y en los que tenían niveles más altos de hemoglobina $(\mathrm{p}=0.0412)$. El SCR1 se asoció a una mayor mortalidad intrahospitalaria a 30 días (HR: 4.11; IC95\%: 1.20-14.09; $p=0.0244)$.

Conclusiones: La incidencia de SCR1 en la unidad de cuidados intensivos coronarios encontrada en nuestro estudio es similar a la descrita en estudios extranjeros. La presencia de shock cardiogénico como causa de falla cardíaca y valores más altos de hemoglobina se asociaron a una mayor incidencia de SCR1. Los pacientes con SCR1 tuvieron mayor mortalidad intrahospitalaria a 30 días.

(c) 2014 Instituto Nacional de Cardiología Ignacio Chávez. Publicado por Masson Doyma México S.A. Todos los derechos reservados.
\end{abstract}

\footnotetext{
* Autor para correspondencia. Av. Paseo de la República 3937 departamento 502 Surquillo, Lima Perú. Tel.:éfono: +511 2212555, celular 51987410911.

Correo electrónico: paulmprezac@hotmail.com (P.M. Preza).
} 


\section{KEYWORDS}

Cardio-renal syndrome; Heart failure; Renal insufficiency; Hospital mortality; Peru

\author{
Cardiorenal syndrome type 1 in the intensive coronary care unit of the Hospital \\ Nacional Arzobispo Loayza
}

\begin{abstract}
Objectives: This study sought to evaluate the incidence of cardiorenal syndrome (CRS) type 1 in a coronary care unit and its association with hospital mortality within 30 days of admission, as well as other epidemiological characteristics.

Methods: The medical records of all the patients who were hospitalized with the diagnosis of acute heart failure in a 4-year period were reviewed. CRS type 1 was characterized by the presence of acute heart failure and an elevation of serum creatinine $\geq 0.3 \mathrm{mg} / \mathrm{dL}$ in comparison to the baseline creatinine calculated by the MDRD75 equation and/or the elevation of $\geq 50 \%$ of the admission serum creatinine within a $48 \mathrm{~h}$ period.

Results: The incidence of CRS type 1 was $27.87 \%, 95 \% \mathrm{Cl}: 20.13-36.71$ (34 of 122). There was a higher frequency of CRS type 1 in those patients who were admitted with the diagnosis of cardiogenic shock (adjusted RR 2.02, 95\% Cl: 1.20-3.93, $\mathrm{p}=0.0378$ ) and in those with higher hemoglobin levels $(p=0.0412)$. The CRS type 1 was associated with an increase of 30 -day mortality (HR: $4.11,95 \% \mathrm{Cl}: 1.20-14.09, \mathrm{p}=0.0244$ ).

Conclusions: The incidence of CRS type 1 in the coronary care unit found in our study is similar to those found in foreign studies. The history of stroke and the higher values of hemoglobin were associated with a higher incidence of cardiorenal syndrome type 1. Patients with CRS type 1 had a higher hospital mortality within 30 days of admission.

(c) 2014 Instituto Nacional de Cardiología Ignacio Chávez. Published by Masson Doyma México S.A. All rights reserved.
\end{abstract}

\section{Introducción}

La falla cardíaca o insuficiencia cardíaca (IC) es uno de los principales problemas de salud pública tanto en países desarrollados como en países en vías de desarrollo y una de las enfermedades mundiales más prevalentes y de mayor mortalidad $^{1-5}$. Esta es definida como un síndrome clínico producido por cualquier disfunción cardíaca, funcional o estructural, que altere la capacidad del ventrículo para el llenado o la eyección sanguínea ${ }^{6}$.

La prevalencia de la IC se sitúa en el 2-3\% y aumenta drásticamente alrededor de los 75 años de edad, hasta llegar a un $10-20 \%$ en el grupo de pacientes de $70-80$ años $^{7}$.

En nuestro país se han hecho estudios analizando las características epidemiológicas de los pacientes hospitalizados por falla cardíaca y su mortalidad intrahospitalaria puede llegar incluso al $17 \%{ }^{8}$. Aproximadamente el $25-33 \%$ de los pacientes que se hospitalizan por falla cardíaca aguda desarrollan enfermedad renal aguda ${ }^{9}$. A esta secuencia de falla cardíaca aguda seguida de falla renal aguda se le conoce en la actualidad como síndrome cardiorrenal agudo o síndrome cardiorrenal tipo 1 (SCR1), el cual es uno de los 5 subtipos de síndrome cardiorrenal según la clasificación propuesta en el 7. ${ }^{\circ}$ consenso de la «Acute Dialysis Quality Initiative» $(A D Q I)^{10}$ (tabla 1). Sin embargo, no existen estudios en nuestro país sobre la incidencia de este síndrome ni sus características epidemiológicas asociadas.

El objetivo de este estudio fue evaluar la incidencia de SCR1 en los pacientes que ingresaron por falla cardíaca aguda a una unidad de cuidados intensivos coronarios (UClCOR) y su asociación a mortalidad intrahospitalaria a 30 días. También se evaluó si existía mayor incidencia de SCR1 asociado a antecedentes (por ejemplo, infarto, accidente cerebrovascular [ACV], insuficiencia cardíaca crónica, etc.), tratamiento previo, tipo de falla cardíaca aguda y asociación con ciertos parámetros de laboratorio. Por último se evaluó si la presencia de SCR1 determinaba cambios en la terapéutica, tiempo de hospitalización o necesidad de diálisis.

\section{Métodos}

Se trata de un estudio observacional, analítico, de cohortes retrospectivas. Se analizaron todas las historias clínicas de los pacientes que ingresaron con diagnóstico de falla cardíaca aguda a la UCICOR del Hospital Nacional Arzobispo Loayza en Lima-Perú, entre las fechas de abril del 2009 y marzo del 2013 según el cuaderno de registro de pacientes de esta unidad.

Los criterios de inclusión para este estudio fueron: diagnóstico de falla cardíaca aguda según criterios $A+B+C$ mencionados más adelante, edad mayor o igual a 18 años y pacientes hospitalizados en la UCICOR del Hospital Nacional Arzobispo Loayza durante el periodo de estudio. Los criterios de exclusión fueron: tamaño longitudinal renal $<9 \mathrm{~cm}$ y/o parénquima $<15 \mathrm{~mm}$ por ecografía, paciente en diálisis, paciente con diagnóstico establecido de insuficiencia renal crónica por historia clínica, hemoglobina $<10 \mathrm{mg} / \mathrm{dL}$ al ingreso, diagnóstico de shock séptico, creatinina previa a la basal (en historia clínica antigua) $>1,5 \mathrm{mg} / \mathrm{dL}$, pacientes recibiendo quimioterapia, falta de creatinina a la admisión, prohormona del péptido natriurético cerebral $<125 \mathrm{pg} / \mathrm{mL}$ durante las primeras $48 \mathrm{~h}$ y pacientes transferidos a otro hospital dentro de los primeros 30 días desde su ingreso. 
Tabla 1 Clasificación del síndrome cardiorrenal

\begin{tabular}{|c|c|c|}
\hline Tipo & Nomenclatura & Definición \\
\hline 1 & Síndrome cardiorrenal agudo & Deterioro agudo de la función cardíaca que conduce a disfunción renal \\
\hline 2 & Síndrome cardiorrenal crónico & Anormalidades crónicas en la función cardíaca que conducen a disfunción renal \\
\hline 3 & Síndrome renocardíaco agudo & Deterioro agudo de la función renal que conduce a disfunción cardíaca \\
\hline 4 & Síndrome renocardíaco crónico & $\begin{array}{l}\text { Anormalidades crónicas en la función renal que conducen a enfermedad } \\
\text { cardíaca }\end{array}$ \\
\hline 5 & Síndrome cardiorrenal secundario & $\begin{array}{l}\text { Condiciones sistémicas que causan simultáneamente disfunción del corazón y } \\
\text { el riñón }\end{array}$ \\
\hline
\end{tabular}

Fuente: Ronco C, McCullough P, Anker SD, et al. Cardio-renal syndromes: Report from the consensus conference of the Acute Dialysis Quality Initiative. Eur Heart J. 2010;31:703-711.

Para confirmar el diagnóstico de falla cardíaca aguda todo paciente debía cumplir con los ítems $A+B+C$ que se muestran a continuación:

A) Presencia de por lo menos 2 criterios mayores o uno mayor y 2 menores según los criterios de Framingham para falla cardíaca (fig. 1) $)^{11,12}$,

B) Criterios ecocardiográficos de falla cardíaca definidos como la presencia de por lo menos una de las siguientes características $^{13-18}$ :
- Disfunción sistólica: fracción de eyección $\leq 50 \%$

- Disfunción diastólica tipo I, II 0 III (tabla 2)

- Si el patrón de llenado es monofásico por ecocardiografía: presencia de e' $\leq 8 \mathrm{~cm} / \mathrm{seg}$ y/o E/e' $>15$

- Si el paciente no cuenta con ecocardiografía o si E/e' está entre 8 y 15: ausencia de alguna otra enfermedad que explique mejor los signos y síntomas que sugieren falla cardíaca ${ }^{3,19}$. (Por ejemplo, paciente disneico por crisis asmática, con miembros inferiores aumentados de volumen por trombosis venosa o celulitis, etc.)

Criterios de inclusión:

\begin{tabular}{|l|l|l|l|}
\hline \multicolumn{2}{|c|}{ Criterios de framingham para insuficincia cardiaca } \\
\hline Dísnea paroxística nocturna & & \multicolumn{2}{c|}{ Edema de los miembros inferiores } \\
\hline Ingurgitación Yugular & & Tos nocturna \\
\hline Estertores & & Disnea de Esfuerzo \\
\hline Cardiomegalia Radiografica & & Hepatomegalia \\
\hline Edema Agudo de Pulmon & & Derrame Pleural \\
\hline Galope por tercer ruido & & $\begin{array}{l}\text { Disminución de la capacidad vital a 1/3 de la } \\
\text { máxima registrada }\end{array}$ \\
\hline Reflujo Hepatoyugular & & $\begin{array}{l}\text { Taquicardia (frecuencia cardiaca }>120 \text { latidos por } \\
\text { minuto) }\end{array}$ \\
\hline Pérdida de peso $>4,5 \mathrm{Kg}$ en 5 días sin tto & & & \\
\hline
\end{tabular}

Cumple criterios de falla cardiaca aguda $\mathrm{A}+\mathrm{B}+\mathrm{C}$ del protocolo

(si) (no)

Tiene 18 años de edad o mas

(si) (no)

Se hospitalizó en la UCI COR

(si) (no)

Criterios de exclusión:

Ecografía: tamaño renal menor de $9 \mathrm{~cm}$ y/o parénquima menor de $15 \mathrm{~mm}$

(si) (no)

Paciente en diálisis

(si) (no)

Paciente con diagnostico ya establecido de IRC

(si) (no)

Creatinina previa a la basal (en historia clínica antigua) $>1,5 \mathrm{mg} / \mathrm{dl}$

(si) (no)

Pacientes recibiendo quimioterapia

(si) (no)

Falta de creatinina a la admisión

(si) (no)

Probnp durante las primeras $48 \mathrm{~h}<125 \mathrm{pg} / \mathrm{ml}$

(si) (no)

Hemoglobina menor a $10 \mathrm{mg} / \mathrm{dl}$

(si) (no)

Diagnostico de shock séptico

(si) (no)

Pacientes transferidos a otro hospital dentro de los 30 días

(si) (no)

Figura 1 Criterios de Framingham para insuficiencia cardíaca y criterios de inclusión y exclusión. 
Tabla 2 Grados de disfunción diastólica y su definición por ecocardiografía transtorácica

\begin{tabular}{lll}
\hline Tipo & Definición & Relación E/A \\
\hline No disfunción & Normal & $\geq 1 \mathrm{y} \leq 2$ \\
Disfunción diastólica tipo I & Relajación anormal o relajación prolongada & $\mathrm{E} / \mathrm{A}<1$ \\
Disfunción diastólica tipo II & Patrón seudonormalizado & $\geq 1 \mathrm{y} \leq 2$ \\
& & Cambio de patrón normal a relajación \\
& & prolongada (con disminución en la E/A $\geq 0.5)$ \\
Disfunción diastólica tipo III & Patrón restrictivo & E/A $>2$ \\
\hline
\end{tabular}

E/A: cociente de los valores de las ondas $\mathrm{E}$ y A del flujo transmitral medidos por doppler pulsado.

Extraída de McMurray et al. $^{3}$.

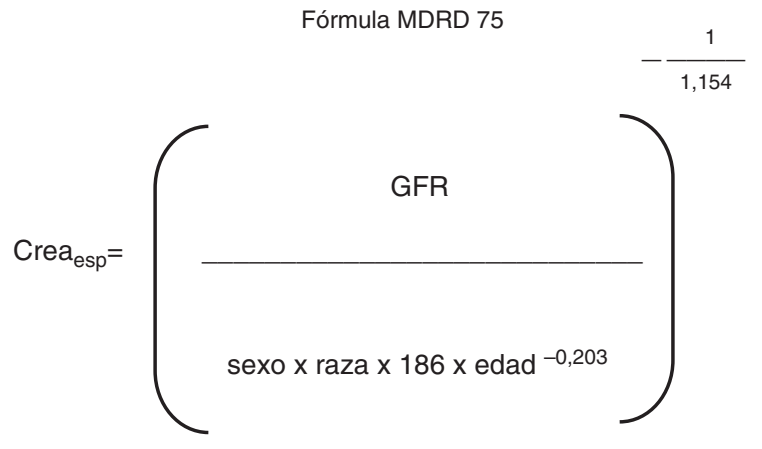

GFR = es el índice de filtración glomerular asumido (que para este estudio será $75 \mathrm{~mL} / \mathrm{min} / \mathrm{m} 2)$

Sexo $=1$ si es varón y 0.742 si es mujer

Raza $=1,21$ si es negro y 1 si es cualquier otro tipo

Edad $=$ en años

Crea $_{\text {esp }}=$ creatinina esperada (que servirá de creatinina basal para este estudio)

Figura 2 Fórmula MDRD75.

C) Inicio o empeoramiento de disnea, palpitaciones o edemas que motive la hospitalización o que haga plantear el diagnóstico de falla cardíaca aguda ${ }^{20,21}$.

Entre los pacientes con falla cardíaca aguda se definió como SCR1 a una elevación de la creatinina $\geq 0.3 \mathrm{mg} / \mathrm{dL}$ $(\geq 0.3 \mathrm{mg} / \mathrm{dL})$ con respecto a la creatinina basal calculada y/o una elevación $\geq 50 \%$ en un periodo de $48 \mathrm{~h}$ (1.5 veces el basal medido en el primer examen de laboratorio al ingreso) ${ }^{22,23}$. Se tomó como creatinina basal calculada un valor derivado de la fórmula MDRD (Modification of Diet in Renal Disease) a partir de un índice de filtración glomerular esperado de $75 \mathrm{~mL} / \mathrm{min} / \mathrm{m}^{2}$ (fig. 2). Se eligió este método para calcular la creatinina basal debido a que la mayoría de los pacientes que llegan al hospital no cuentan con una creatinina previa y en múltiples estudios este problema fue enfrentado calculando una creatinina basal a partir de la fórmula de depuración de creatinina (en este caso la fórmula MDRD) tomando como constante una presunta función basal de $75 \mathrm{~mL} / \mathrm{min} / \mathrm{m}^{2}$ (MDRD75), tal como lo recomienda la ADQI Working Group ${ }^{24-26}$. Este valor de filtración glomerular representa la peor función renal basal posible en una persona sin falla renal, en lugar de una presunta función renal basal entre 80 y 120 (que son sus valores normales).
De esta forma se disminuye el número de diagnósticos falsos positivos.

Para la recolección de datos se utilizó un formulario (fig. 3) supervisado por los coinvestigadores nefrólogos y cardiólogos y aprobado por el Comité Institucional de Ética de la Universidad Peruana Cayetano Heredia. Las variables independientes utilizadas fueron: sexo; edad; antecedentes de insuficiencia cardíaca crónica, hipertensión arterial, diabetes mellitus, accidente cerebrovascular (ACV), valvulopatía severa, infarto de miocardio, angiografía coronaria y arritmia; tipo de arritmia que puede incluir fibrilación auricular, flutter, taquicardia paroxística supraventricular, bloqueo auriculoventricular y otras; medicación previa considerada en la historia clínica de ingreso de la UCICOR: inhibidores de la enzima convertidora de la angiotensia, antagonistas de los receptores de la angiotensina II, diuréticos, betabloqueadores, espironolactona, calcioantagonistas, ácido acetilsalicílico, nitratos, antiinflamatorios no esteroideos y digitálicos; tipo de IC aguda: insuficiencia cardíaca crónica descompensada, edema agudo de pulmón, insuficiencia cardíaca hipertensiva, shock cardiogénico, IC derecha aislada e IC asociada a síndrome isquémico coronario agudo; valores de algunos exámenes auxiliares al ingreso como: sodio, potasio, bicarbonato, hemoglobina, lactato, diuresis durante las primeras $24 \mathrm{~h}$ y diuresis entre las 24 y $48 \mathrm{~h}$; fracción de eyección por ecocardiografía; función diastólica por ecocardiografía; síndrome cardiorrenal. Las variables dependientes usadas fueron: síndrome cardiorrenal; uso de fármacos durante las primeras $48 \mathrm{~h}$ como: inhibidores de la enzima convertidora de angiotensia, antagonistas de los receptores de la angiotensina ॥, furosemida, betabloqueadores, espironolactona, antiinflamatorios no esteroideos, calcioantagonistas, ácido acetilsalicílico, nitratos, inotrópicos, vasopresores, digitálicos, fibrinolíticos, ranitidina, hidroclorotiazida, enoxaparina, warfarina y clopidogrel; tiempo de hospitalización; diálisis; mortalidad intrahospitalaria a 30 días (tabla 3).

Los exámenes de sangre registrados fueron tomados en el laboratorio del Hospital, el cual utiliza el analizador de gases en sangre $A B L 800$ FLEX para medir sodio, potasio, bicarbonato y lactato; el analizador fotométrico COBAS C501 ROCHE para medir la creatinina en sangre y el sistema hematológico ADVIA 2120 para analizar la hemoglobina.

Para todas la ecocardiografías se utilizó ecógrafos Phillips iE33.

Se utilizó T de Student para variables continuas, $\mathrm{chi}^{2}$ para variables cualitativas y análisis de regresión de Cox para 
Formulario "sindrome cardiorenal tipo i en la unidad de cuidados intensivos coronarios del hospital nacional arzobispo loayza"

Datos personales
Antecedentes: (Marcar con X)
\begin{tabular}{|l|l|l|l|l|l|l|l|l|}
\hline & ICC & HTA & DM & ACV & VALV & IMA & CATE & Arritmia \\
\hline SI & & & & & & & & \\
\hline NO & & & & & & & & \\
\hline
\end{tabular}

Si arritmia es si $\rightarrow$ Tipo de arritmia: FA FLUTTER TPSV BAV Otras

Otros antecedentes:

Medicacion previa
\begin{tabular}{|l|l|l|}
\hline \multicolumn{1}{|c|}{} & SI & NO \\
\hline IECA & & \\
\hline ARA II & & \\
\hline Diureticos & & \\
\hline BBLOQ & & \\
\hline Espironolactona & & \\
\hline
\end{tabular}

\begin{tabular}{|l|l|l|}
\hline & SI & NO \\
\hline Calcioantagonitas & & \\
\hline AAS & & \\
\hline Nitratos & & \\
\hline AINES & & \\
\hline Digitalicos & & \\
\hline
\end{tabular}

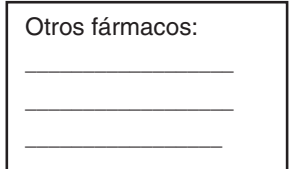

Enfermedad actual: Tiempo de enfermedad en dias :

Peso:

Talla:

Causa o tipo de insuficiencia cardíaca aguda:

\begin{tabular}{|l|l|l|l|l|l|l|}
\hline & $\begin{array}{l}\text { ICC } \\
\text { Descompensada }\end{array}$ & EAP & $\begin{array}{l}\text { IC } \\
\text { Hipertensiva }\end{array}$ & $\begin{array}{l}\text { Shock } \\
\text { Cardiogénico }\end{array}$ & $\begin{array}{l}\text { IC Derecha } \\
\text { Aislada }\end{array}$ & $\begin{array}{l}\text { IC asociada a } \\
\text { SICA }\end{array}$ \\
\hline SI & & & & & & \\
\hline NO & & & & & & \\
\hline
\end{tabular}

Otras causas de ic aguda:

Si la causa es ic cronica descompensada: ¿cuál es la causa de descompensación?

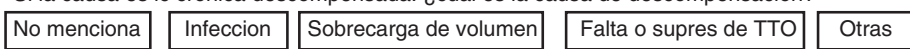

Examenes auxiliares

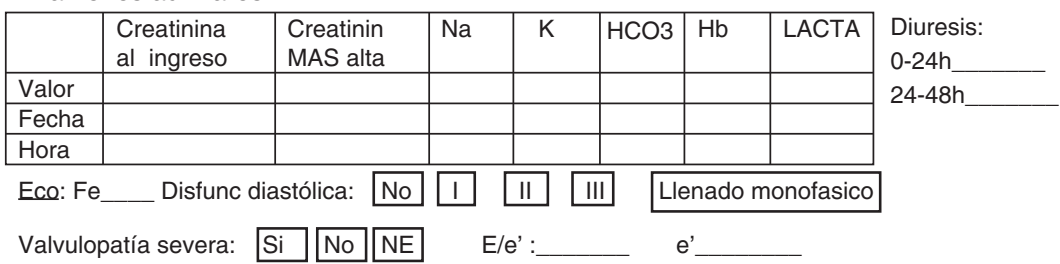

Terapéutica utilizada en las primeras $48 \mathrm{~h}$

\begin{tabular}{|l|l|l|}
\hline & SI & NO \\
\hline IECA & & \\
\hline BBLOQ & & \\
\hline Ca ANT & & \\
\hline Inotrópico & & \\
\hline Fibrinolitico & & \\
\hline Enoxaparina & & \\
\hline
\end{tabular}

\begin{tabular}{|l|l|l|}
\hline & SI & NO \\
\hline ARA II & & \\
\hline Espironolactona & & \\
\hline AAS & & \\
\hline Vasopresor & & \\
\hline Ranitidina & & \\
\hline Warfarina & & \\
\hline
\end{tabular}

\begin{tabular}{|l|l|l|}
\hline & SI & NO \\
\hline Furosemida & & \\
\hline AINE & & \\
\hline Nitrato & & \\
\hline Digital & & \\
\hline HCTZ & & \\
\hline Clopidogrel & & \\
\hline
\end{tabular}

Otros tratamientos:

Evolución

Se realizó dialisis drante la hospitalización: Si No

Fecha de alta:

Falleció durante la hospitalización: Si No

Si el paciente falleció entonces llenar lo siguiente

Fecha de fallecimiento: (Igual a fecha del alta)

Motivo de fallecimiento:

Figura 3 Formulario para la recolección de datos. 
Tabla 3 Tipos de variables y sus posibles valores

\begin{tabular}{|c|c|c|}
\hline Nombre & Tipo & Valores \\
\hline Sexo & Dico & $M / F$ \\
\hline Edad en años & Cuant & 18 a más \\
\hline Antecedente de ICC & Dico & Sí/No \\
\hline Antecedente de HTA & Dico & Sí/No \\
\hline Antecedente de DM & Dico & Sí/No \\
\hline Antecedente de ACV & Dico & Sí/No \\
\hline $\begin{array}{l}\text { Antecedente de valvulopatía } \\
\text { severa }\end{array}$ & Dico & Sí/No \\
\hline Antecedente de IM & Dico & Sí/No \\
\hline $\begin{array}{l}\text { Cateterismo cardíaco } \\
\text { (angiografía coronaria } \\
\text { reciente) }\end{array}$ & Dico & Sí/No \\
\hline Arritmia & Cual & $\begin{array}{l}\text { FA, flutter, } \\
\text { TPSV, BAV, } \\
\text { otras }\end{array}$ \\
\hline \multicolumn{3}{|l|}{ Medicación previa } \\
\hline IECA & Dico & Sí/No \\
\hline ARAII & Dico & Sí/No \\
\hline Diuréticos & Dico & Sí/No \\
\hline Betabloqueadores & Dico & Sí/No \\
\hline Espironolactona & Dico & Sí/No \\
\hline Calcioantagonistas & Dico & Sí/No \\
\hline AAS & Dico & Sí/No \\
\hline Nitratos & Dico & Sí/No \\
\hline AINE & Dico & Sí/No \\
\hline Digitálicos & Dico & Sí/No \\
\hline $\begin{array}{l}\text { Tiempo de enfermedad en días } \\
\text { Tipo de falla cardíaca aguda }\end{array}$ & Cuant & 0 a más \\
\hline IC crónica descompensada & Dico & Sí/No \\
\hline Edema agudo de pulmón & Dico & Sí/No \\
\hline IC hipertensiva & Dico & Sí/No \\
\hline Shock cardiogénico & Dico & Sí/No \\
\hline IC derecha aislada & Dico & Sí/No \\
\hline IC asociada a SICA & Dico & Sí/No \\
\hline Na en $\mathrm{mEq} / \mathrm{L}$ & Cuanti & 0 a más \\
\hline$K$ en $\mathrm{mEq} / \mathrm{L}$ & Cuanti & 0 a más \\
\hline $\mathrm{HCO}_{3}$ en $\mathrm{mEq} / \mathrm{L}$ & Cuanti & 0 a más \\
\hline$H b$ en $m g / d L$ & Cuanti & 0 a más \\
\hline Lactato en $\mathrm{mEq} / \mathrm{L}$ & Cuanti & 0 a más \\
\hline Diuresis(primeras $24 h$ ) en $\mathrm{mL}$ & Cuanti & 0 a más \\
\hline Diuresis(24 a $48 h$ ) en $m L$ & Cuanti & 0 a más \\
\hline FE en $\%$ & Cuanti & 0 a 100 \\
\hline Función diastólica & Cuali & $\begin{array}{l}\text { Disfunción tipo } \\
\text { I, II, III y patrón } \\
\text { monofásico }\end{array}$ \\
\hline \multicolumn{3}{|c|}{ Fármacos usados en las primeras $48 \mathrm{~h}$} \\
\hline IECA & Dico & Sí/No \\
\hline ARAII & Dico & Sí/No \\
\hline Furosemida & Dico & Sí/No \\
\hline Betabloqueadores & Dico & Sí/No \\
\hline Espironolactona & Dico & Sí/No \\
\hline AINE & Dico & Sí/No \\
\hline Calcioantagonistas & Dico & Sí/No \\
\hline AAS & Dico & Sí/No \\
\hline Nitratos & Dico & Sí/No \\
\hline
\end{tabular}

Tabla 3 (continuación)

\begin{tabular}{lll}
\hline Nombre & Tipo & Valores \\
\hline Inotrópicos & Dico & Sí/No \\
Vasopresores & Dico & Sí/No \\
Digitálicos & Dico & Sí/No \\
Fibrinolíticos & Dico & Sí/No \\
Ranitidina & Dico & Sí/No \\
Hidroclorotiazida & Dico & Sí/No \\
Enoxaparina & Dico & Sí/No \\
Warfarina & Dico & Sí/No \\
Clopidogrel & Dico & Sí/No \\
Tiempo de hospitalización en & Cuanti & 0 a más \\
días & & \\
Diálisis & Dico & Sí/No \\
Mortalidad a 30 días & Dico & Sí/No \\
\hline
\end{tabular}

AAS: ácido acetilsalicílico; ACV: accidente cerebrovascular; AINE: antiinflamatorios no esteroideos; ARAll: antagonista de los receptores de la angiotensina ॥; BAV: bloqueo auriculoventricular de alto grado; Cual: cualitativa; Cuanti: cuantitativa; Dico: dicotómica; DM: diabetes mellitus; F: femenino; FA: fibrilación auricular; FE: fracción de eyección; $\mathrm{Hb}$ : hemoglobina; $\mathrm{HCO}_{3}$ : bicarbonato; HTA: hipertensión arterial; IC: insuficiencia cardíaca; ICC: insuficiencia cardíaca crónica; IECA: inhibidores de la enzima convertidora de la angiotensina; IM: infarto de miocardio; K: potasio; M: masculino; Na: sodio; SICA: síndrome isquémico coronario agudo; TPSV: taquicardia paroxística supraventricular.

calcular la supervivencia. El programa utilizado para realizar el análisis estadístico de los resultados fue Epi Info ${ }^{T M}$ 7.1.2.0.

\section{Resultados}

Se analizaron un total de 167 historias clínicas, de las cuales solo 122 cumplían con criterios de inclusión y exclusión. Los motivos para excluir a los pacientes en orden de frecuencia fueron: 19 por no cumplir criterios de falla cardíaca aguda, 5 por riñones menores de $90 \mathrm{~mm}$ por ecografía, 5 por enfermedad renal crónica (ERC) previa según criterio del médico que recibió al paciente en su ingreso a la UCICOR, 4 por shock séptico, 3 por ausencia de creatinina al ingreso, 3 por diagnóstico de ERC en hemodiálisis, 2 por otras causas que pueden explicar signos y síntomas que asemejen IC ante la ausencia de confirmación ecocardiográfica, uno por ser paciente menor de 18 años de edad, uno por ser transferido antes del alta, uno por creatinina previa $>1.5$ y uno por hemoglobina al ingreso $<10 \mathrm{mg} / \mathrm{dL}$.

De los 122 pacientes que ingresaron al estudio, 34 (27.87\%) tuvieron el diagnóstico de SCR1 con un IC $95 \%$ 20.13-36.71\%. Las características clínicas de los pacientes se muestran en la tabla 4. No hubo diferencias significativas en sexo, edad, medicación previa, tiempo de enfermedad (tiempo desde que comenzaron los síntomas hasta su ingreso), ni grado ecocardiográfico de disfunción sistólica o diastólica. En el análisis univariado tanto el ACV como antecedente y el shock cardiogénico como causa de falla cardíaca aguda estuvieron asociados a un mayor 
Tabla 4 Análisis de las variables independientes y su asociación a SCR1

\begin{tabular}{|c|c|c|c|c|c|c|}
\hline Variable & $\begin{array}{l}\text { Todos } \\
\mathrm{N}=122\end{array}$ & $\begin{array}{l}\text { Con SCR } \\
N=34\end{array}$ & $\begin{array}{l}\text { Sin SCR } \\
N=88\end{array}$ & $\mathrm{RR}$ & IC95\% & $\mathrm{p}$ \\
\hline Sexo masculino $\mathrm{n}(\%)$ & $67(54.92)$ & $20(58.82)$ & $47(53.41)$ & 1.17 & $0.66-2.10$ & 0.6861 \\
\hline Sexo femenino, n (\%) & $55(45.08)$ & $14(41.18)$ & $41(46.59)$ & & & \\
\hline Edad, $\boldsymbol{\mu} \pm \boldsymbol{\sigma}$ & $64.89 \pm 15.97$ & $68.24 \pm 14.04$ & $63.60 \pm 16.55$ & - & - & 0.1517 \\
\hline \multicolumn{7}{|l|}{ Antecedentes } \\
\hline Insuficiencia cardíaca crónica, n (\%) & $49(40.16)$ & $12(35.29)$ & $37(42.05)$ & 0.81 & $0.44-1.49$ & 0.5420 \\
\hline Hipertensión arterial, $n$ (\%) & $51(41.8)$ & $15(44.12)$ & $36(40.91)$ & 1.10 & $0.62-1.95$ & 0.8384 \\
\hline Diabetes mellitus, $n(\%)$ & $23(18.85)$ & $8(23.53)$ & $15(17.05)$ & 1.32 & $0.69-2.54$ & 0.4438 \\
\hline Accidente cerebrovascular, $n(\%)$ & $11(9.02)$ & $7(20.59)$ & $4(4.55)$ & 2.09 & $1.23-3.56$ & 0.0587 \\
\hline Valvulopatía, $n(\%)$ & $22(18.03)$ & $6(17.65)$ & $16(18.18)$ & 0.97 & $0.46-2.06$ & 1.0000 \\
\hline Infarto de miocardio, $n(\%)$ & $19(15.57)$ & $5(14.71)$ & $14(14.77)$ & 0.93 & $0.42-2.11$ & 1.0000 \\
\hline Cateterismo cardíaco, $n(\%)$ & $7(5.74)$ & $2(5.88)$ & $5(5.68)$ & 1.03 & $0.31-3.44$ & 1.0000 \\
\hline Arritmia, $n(\%)$ & $34(27.87)$ & $12(35.29)$ & $22(25)$ & 1.41 & $0.79-2.53$ & 0.2680 \\
\hline Fibrilación auricular, n & 23 & 8 & 15 & - & - & - \\
\hline Flutter, $\mathrm{n}$ & 2 & 0 & 2 & & - & - \\
\hline TPSV, $\mathrm{n}$ & 3 & 1 & 2 & & - & - \\
\hline $\mathrm{BAV}, \mathrm{n}$ & 6 & 3 & 3 & & - & - \\
\hline \multicolumn{7}{|l|}{ Medicación previa, n (\%) } \\
\hline IECA & $34(27.87)$ & $8(23.53)$ & $26(29.55)$ & 0.80 & $0.40-1.58$ & 0.6533 \\
\hline ARAII & $11(9.02)$ & $5(14.71)$ & $6(6.82)$ & 1.74 & $0.85-3.57$ & 0.1783 \\
\hline Diuréticos & $31(25.41)$ & $9(26.47)$ & $22(25)$ & 1.06 & $0.56-2.01$ & 1.0000 \\
\hline Betabloqueadores & $13(10.66)$ & $4(11.76)$ & $9(10.23)$ & 1.12 & $0.47-2.67$ & 0.7541 \\
\hline Espironolactona & $23(18.85)$ & $6(17.65)$ & $17(19.32)$ & 0.92 & $0.43-1.96$ & 1.0000 \\
\hline Calcioantagonistas & $8(6.56)$ & $2(5.88)$ & $6(6.82)$ & 0.89 & $0.26-3.06$ & 1.0000 \\
\hline Ácido acetilsalicílico & $36(29.51)$ & $6(17.65)$ & $30(34.09)$ & 0.51 & $0.23-1.29$ & 0.0815 \\
\hline Nitratos & $12(9.84)$ & $5(14.71)$ & $7(7.95)$ & 1.58 & $0.76-3.31$ & 0.3119 \\
\hline AINE & $1(0.82)$ & $0(0)$ & $1(1.14)$ & - & - & 1.0000 \\
\hline Digitálicos & $21(17.21)$ & $4(11.76)$ & $17(19.32)$ & 0.64 & $0.25-1.63$ & 0.4267 \\
\hline Tiempo de enfermedad, $\boldsymbol{\mu} \pm \boldsymbol{\sigma}$ & $10.39 \pm 19.58$ & $9.57 \pm 16.57$ & $10.71 \pm 20.70$ & - & - & 0.7757 \\
\hline \multicolumn{7}{|l|}{ Tipo de IC aguda, n (\%) } \\
\hline IC crónica descompensada & $76(62.30)$ & $20(58.82)$ & $56(63.64)$ & 0.86 & $0.49-1.54$ & 0.6792 \\
\hline Edema agudo de pulmón & $17(13.93)$ & $6(17.65)$ & $11(12.50)$ & 1.42 & $0.70-2.88$ & 0.3779 \\
\hline IC hipertensiva & $6(4.92)$ & $2(5.88)$ & $4(4.55)$ & 1.21 & $0.38-3.89$ & 0.6705 \\
\hline Shock cardiogénico & $18(14.75)$ & $10(29.41)$ & $8(9.09)$ & 2.02 & $1.20-3.93$ & 0.0378 \\
\hline IC derecha aislada & $4(3.28)$ & $1(2.94)$ & $3(3.41)$ & 0.89 & $0.16-5.00$ & 1.0000 \\
\hline IC asociada a SICA & $31(25.41)$ & $8(23.53)$ & $23(26.14)$ & 0.90 & $0.46-1.78$ & 0.8210 \\
\hline \multicolumn{7}{|l|}{ Exámenes auxiliares } \\
\hline $\mathrm{Na}, \mu \pm \sigma$ & $136.61 \pm 6.17$ & $135.77 \pm 6.04$ & $136.93 \pm 6.22$ & - & - & 0.3671 \\
\hline$K, \mu \pm \sigma$ & $3.91 \pm 0.68$ & $4.09 \pm 0.78$ & $3.84 \pm 0.63$ & - & - & 0.0712 \\
\hline $\mathrm{HCO}_{3}, \mu \pm \sigma$ & $21.13 \pm 8.26$ & $19.03 \pm 5.09$ & $21.95 \pm 9.10$ & - & - & 0.0893 \\
\hline$H b, \mu \pm \sigma$ & $13.14 \pm 1.85$ & $13.68 \pm 1.99$ & $12.91 \pm 1.76$ & - & - & 0.0412 \\
\hline Lactato, $\mu \pm \sigma$ & $2.03 \pm 1.89$ & $2.94 \pm 3.00$ & $1.67 \pm 1.03$ & - & - & 0.0802 \\
\hline Diuresis(primeras $24 \mathrm{~h}), \mu$ & 1,653 & 1,522 & 1,704 & - & - & 0,3557 \\
\hline Diuresis(24-48h), $\mu$ & 1,908 & 1,975 & 1,884 & - & - & 0.6797 \\
\hline Ecografías disponibles & $\mathrm{n}=94$ & $\mathrm{n}=26$ & $\mathrm{n}=68$ & - & - & - \\
\hline FE en $\%, \mu$ & $42.76 \pm 15.79$ & $40.47 \pm 15.21$ & $43.68 \pm 16.03$ & - & - & 0.3488 \\
\hline Función diastólica & - & - & - & - & - & 0.2588 \\
\hline No disfunción, $\mathrm{n}$ & 9 & 0 & 9 & & & \\
\hline Tipo I, n & 33 & 10 & 23 & & & \\
\hline Tipo II, dxzn & 12 & 5 & 7 & & & \\
\hline Tipo III, n & 22 & 5 & 17 & & & \\
\hline Llenado monof, $\mathrm{n}$ & 18 & 6 & 12 & & & \\
\hline
\end{tabular}

AINE: antiinflamatorios no esteroideos; ARAll: antagonista de los receptores de la angiotensina ॥; BAV: bloqueo auriculoventricular de alto grado; FE: fracción de eyección; $\mathrm{Hb}$ : hemoglobina; $\mathrm{HCO}_{3}$ : bicarbonato; IC: insuficiencia cardíaca; IC95\%: intervalo de confianza del 95\%; IECA: inhibidores de la enzima convertidora de la angiotensina; K: potasio; n: frecuencia; Na: sodio; RR: riesgo relativo; SCR: síndrome cardiorrenal; SICA: síndrome isquémico coronario agudo; TPSV: taquicardia paroxística supraventricular; $\mu$ : media; $\sigma$ : desviación estándar; \%: porcentaje.

" Análisis multivariado ajustado para shock cardiogénico y ACV. 
riesgo de desarrollar SCR1 (ACV: RR = 2.62; IC95\% 1.50-4.55; $\mathrm{p}=0.010$ ) (shock cardiogénico: $R R=2.41$; IC95\% 1.40-4.14; $\mathrm{p}=0.0087$ ). Al hacer el análisis multivariado, el shock cardiogénico continuó siendo una variable que significativamente aumenta el riesgo de desarrollar SCR1 (ajustado: $R R=2.02 ; \quad I C 95 \%: 1.20-3.93 ; p=0.0378)$. La asociación a $\mathrm{ACV}$, por otro lado, disminuyó con un valor «p» ligeramente por encima de 0.05 (ajustado: $R R=2.09$; IC95\%: 1.23-3.56; $\mathrm{p}=0.0587$ ).

Niveles más altos de hemoglobina también estuvieron asociados a una mayor incidencia de SCR1 $(p=0.0412)$ en este estudio.

Luego del análisis univariado se encontró que entre los pacientes con SCR1 hubo una mayor utilización de inotrópi$\cos (R R=3.88$; IC95\%: 1.74-8.66; $p=0.0017)$, pero después de hacer el análisis multivariado, corregido por la presencia de shock cardiogénico, esta diferencia no fue estadísticamente significativa (ajustado: RR=1.58; IC95\%: 0.99-2.51; $\mathrm{p}=0.1161$ ) (tabla 5). Por lo tanto, no hubo diferencia estadísticamente significativa para el uso de ninguno de los fármacos evaluados. No se encontró tampoco asociación entre la presencia de SCR1 y el tiempo de hospitalización (media de 5.5 días en pacientes con SCR1 y de 5 días en pacientes sin SCR1; $p=0.6358$ ). Ninguno de los pacientes estudiados fue sometido a diálisis durante su hospitalización.

Los pacientes con SCR1 tuvieron una mayor mortalidad intrahospitalaria a 30 días ( $R R=4.53$; IC95\%: 1.4157 14.4919; $p=0.0105$ ) (tabla 6). De los 34 pacientes que

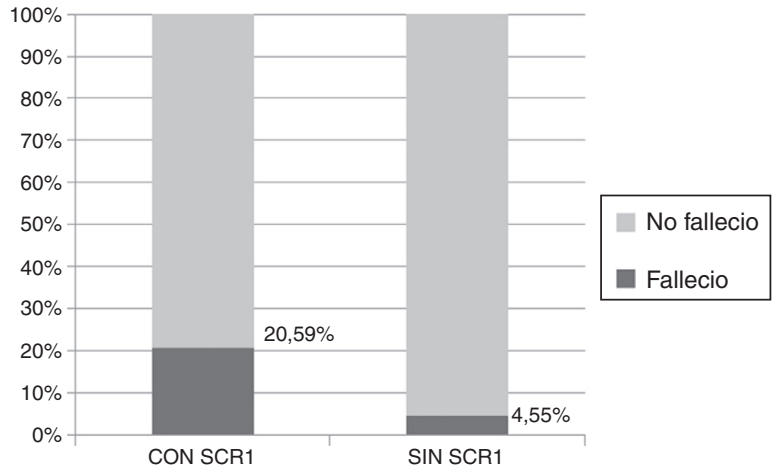

Figura 4 Porcentaje de mortalidad según presencia o ausencia de SCR1.

desarrollaron SCR1 fallecieron 7 (20.59\%) y de los 88 pacientes sin SCR1 fallecieron solo 4 pacientes (4.55\%) (fig. 4). La presencia de shock cardiogénico también estuvo asociada a mayor mortalidad intrahospitalaria a 30 días y debido a que existe una relación causal entre la presencia de shock cardiogénico y el desarrollo de SCR1, pero no en sentido contrario, no se realizó un análisis multivariado corregido por la presencia de shock cardiogénico.

El análisis de regresión de Cox mostró que la mortalidad a 30 días fue mayor entre los pacientes que desarrollaron SCR1 (HR: 4.11; IC95\%: 1.20-14.09; $p=0.0244)$ (fig. 5).

Tabla 5 Análisis de la presencia de SCR1 y su asociación a cambios en la terapéutica en las primeras $48 \mathrm{~h}$, tiempo de hospitalización y necesidad de diálisis

\begin{tabular}{|c|c|c|c|c|c|c|}
\hline & $\begin{array}{l}\text { Total } \\
\mathrm{N}=122\end{array}$ & $\begin{array}{l}\text { Con SCR } \\
\mathrm{N}=34\end{array}$ & $\begin{array}{l}\text { Sin SCR } \\
\mathrm{N}=88\end{array}$ & $\mathrm{RR}$ & IC95\% & $\mathrm{p}$ \\
\hline \multicolumn{7}{|l|}{ Fármacos, $n$ (\%) } \\
\hline IECA & $68(55.74)$ & $15(44.12)$ & $53(60.23)$ & 0.73 & $0.48-0.11$ & 0.1542 \\
\hline ARAII & 2 & 0 & 2 & 1.00 & - & 1.0000 \\
\hline Furosemida & $108(88.52)$ & $30(88.24)$ & $78(88.64)$ & 0.92 & $0.86-1.15$ & 1.0000 \\
\hline Betabloqueadores & $19(15.57)$ & $5(14.71)$ & $14(15.91)$ & 0.63 & $0.36-2.37$ & 1.0000 \\
\hline Espironolactona & $61(50.0)$ & $12(35.29)$ & $49(55.68)$ & 2.59 & $0.39-1.04$ & 0.0683 \\
\hline AINE & 2 & 1 & 1 & 2.59 & $0.17-40.22$ & 0.4814 \\
\hline Calcioantagonistas & $10(8.20)$ & $5(14.71)$ & $5(5.68)$ & 0.79 & $0.80-8.38$ & 0.1394 \\
\hline AAS & $64(52.46)$ & $15(42.12)$ & $49(55.68)$ & 0.75 & $0.52-1.21$ & 0.3131 \\
\hline Nitratos & $31(25.41)$ & 7 (20.59) & $24(27.27)$ & 1.58 & $0.36-1.59$ & 0.4962 \\
\hline Inotrópicos & 20 (16.39) & $12(35.29)$ & $8(9.09)$ & 2.26 & $0.99-2.51$ & $0.1161^{*}$ \\
\hline Vasopresores & $15(12.30)$ & $7(20.59)$ & $8(9.09)$ & 0.91 & $0.89-5.76$ & 0.1211 \\
\hline Digitálicos & $46(37.70)$ & $12(35.29)$ & $34(38.64)$ & - & $0.54-1.55$ & 0.8360 \\
\hline Fibrinolíticos & 0 & $18(52.94)$ & $51(57.95)$ & 0.91 & - & - \\
\hline Ranitidina & $69(56.56)$ & 0 & $2(2.27)$ & - & $0.64-1.31$ & 0.6855 \\
\hline Hidroclorotiazida & $2(1.64)$ & $22(64.71)$ & $60(68.18)$ & 0.95 & - & 1.0000 \\
\hline Enoxaparina & $82(67.21)$ & $4(11.76)$ & $20(22.73)$ & 0.52 & $0.71-1.26$ & 0.8300 \\
\hline Warfarina & $24(19.67)$ & $9(26.47)$ & $32(36.36)$ & 0.73 & $0.19-1.40$ & 0.2108 \\
\hline Clopidogrel & $41(33.61)$ & & & & $0.39-1.36$ & 0.3934 \\
\hline Tiempo de hospitalización (mediana) & 5 & 5.5 & 5 & - & - & 0.6606 \\
\hline Diálisis, $n$ & 0 & 0 & 0 & - & - & - \\
\hline
\end{tabular}

AAS: ácido acetilsalicílico; AINE: antiinflamatorios no esteroideos; ARAll: antagonista de los receptores de la angiotensina ॥; IC95\%: intervalo de confianza del 95\%; IECA: inhibidores de la enzima convertidora de la angiotensina; n: frecuencia; RR: riesgo relativo; SCR: síndrome cardiorrenal; \%: porcentaje.

" Análisis multivariado ajustado para shock cardiogénico. 
Tabla 6 SCR1 y mortalidad intrahospitalaria a 30 días

\begin{tabular}{lcrr}
\hline & Falleció, $\mathrm{n}(\%)$ & No falleció, $\mathrm{n}(\%)$ & Total, $\mathrm{n}(\%)$ \\
\hline Con SCR1 & $7(20.59)$ & $27(79.41)$ & $34(100)$ \\
Sin SCR1 & $4(4.55)$ & $84(95.45)$ & $88(100)$ \\
Total & $11(9.02)$ & $111(90.98)$ & $122(100)$ \\
RR $=(7 / 34) /(4 / 88)=4.53$ & & & \\
IC95\%: $1.4157-14.4919 ; \mathrm{p}=0.0105$ & & & \\
\hline
\end{tabular}

IC95\%: intervalo de confianza del 95\%; RR: riesgo relativo; SCR1: síndrome cardiorrenal tipo 1.

\section{Discusión}

Este estudio evidencia que la incidencia de SCR1 en los pacientes hospitalizados por falla cardíaca aguda en una UCICOR de nuestra ciudad (Lima-Perú) es del 27.87\%; cifra semejante a las obtenidas en estudios extranjeros ${ }^{9,27}$. El shock cardiogénico fue el factor de riesgo más fuertemente asociado al desarrollo de SCR1. El antecedente de ACV fue otro factor de riesgo asociado a desarrollo de SCR1 en el análisis univariado pero que pierde su significación estadística al hacer el análisis multivariado ajustado por la presencia de shock cardiogénico. Sin embargo, estudios previos han demostrado que el antecedente de ACV es un factor de riesgo para desarrollar una caída de la función renal en pacientes que se hospitalizan por falla cardíaca aguda ${ }^{28}$. Por lo tanto no es coincidencia encontrar una mayor incidencia de SCR1 en los pacientes con antecedente de ACV y es posible que su asociación estadística sea más fuerte si tuviéramos una población más numerosa.
Los factores de riesgo que han demostrado mayor incidencia de SCR1 en otros estudios son: insuficiencia cardíaca crónica, diabetes mellitus y presión arterial elevada9,28. Si analizamos en este contexto, la insuficiencia cardíaca crónica y el ACV isquémico, no es difícil de reconocer un factor etiológico común entre ellos: «la aterosclerosis». La diabetes mellitus y la hipertensión arterial son también factores de riesgo asociados al desarrollo de aterosclerosis. Por lo tanto es posible deducir que los pacientes con mayor riesgo de desarrollar SCR1 son aquellos que de alguna forma ya tienen daño endotelial ${ }^{29}$. Por otro lado, el estado de hipoperfusión generado por la falla cardíaca aguda es uno de los factores que precipitan el desarrollo de SCR1 y este estado de hipoperfusión es mucho más notorio en los pacientes que desarrollan shock cardiogénico, entidad que ya ha demostrado también en otros estudios ser una factor importante asociado a SCR $1^{30}$.

En este estudio se evidenció que la presencia de niveles más altos de hemoglobina estuvo asociada a SCR1. Esta

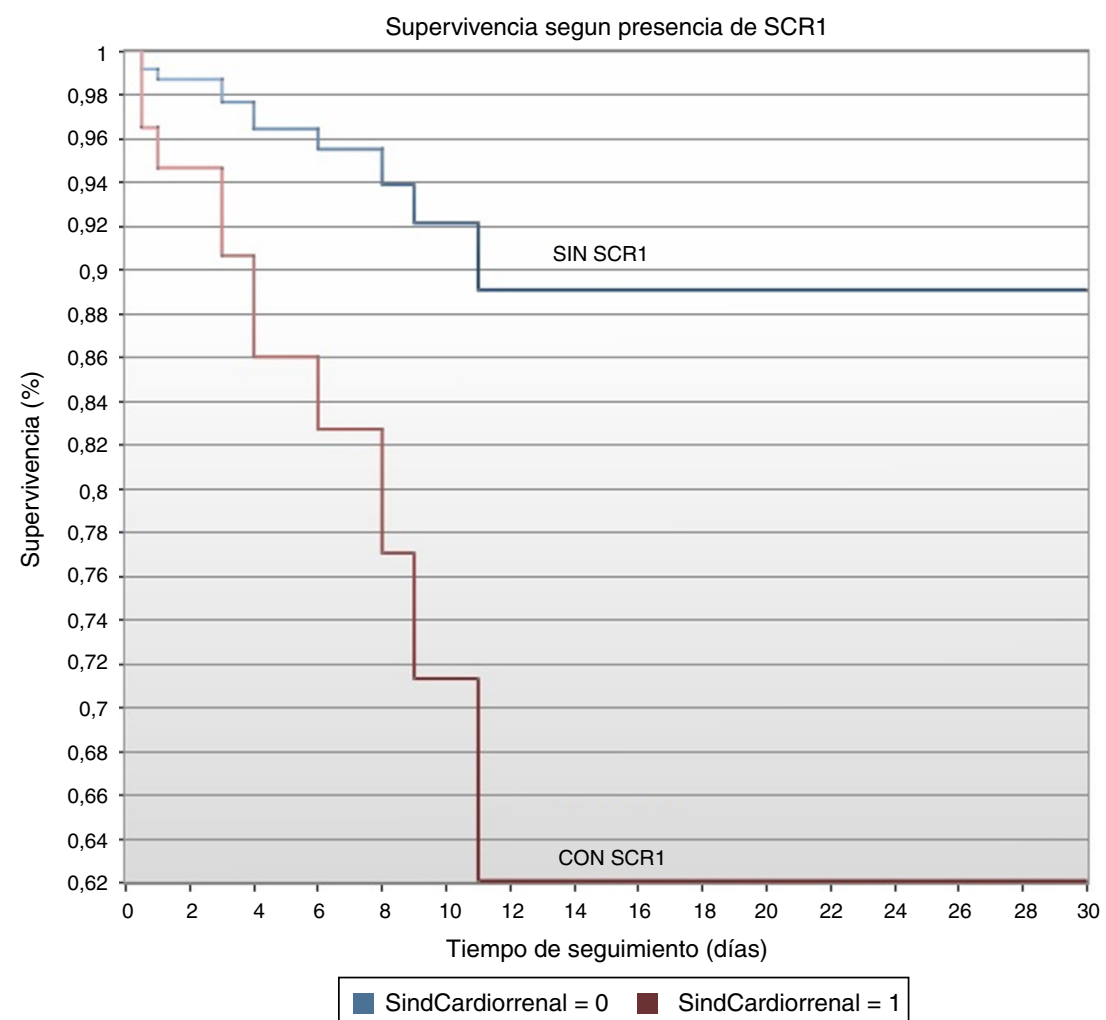

Figura 5 Supervivencia a 30 días según presencia o no de SCR1. 
asociación pudo deberse a que nuestros criterios de inclusión y exclusión estuvieron diseñados para excluir a pacientes con ERC y en este intento se excluyeron 5 historias clínicas de pacientes con creatininas altas y hemoglobinas bajas consideradas en su diagnóstico de ingreso como ERC por el médico que redactó la historia clínica. Sin embargo, en estos casos no se tenían confirmaciones de tamaño renal por ecografía o algún otro dato que confirme que el paciente padecía previamente ERC. Es posible entonces que se hayan excluido pacientes con falla renal aguda que al coexistir con anemia y tener creatininas elevadas fueron consideradas por el médico que recibió al paciente como ERC.

La falta de asociación a cambios en la terapéutica en los pacientes con SCR1 es probablemente debida a que solo se registró la utilización o no utilización de algunos fármacos y no se compararon las dosis utilizadas. Otros estudios han demostrado que la presencia de SCR1 genera en los médicos tratantes cambios en las terapéuticas, como mayor uso de diuréticos ${ }^{31}$ o menor uso de inhibidores de la enzima convertidora de angiotensia ${ }^{32}$. Es posible también que la ausencia de diferencia en el tratamiento se deba a que la enfermedad renal aguda no fue reconocida como tal, ya que el cálculo de la filtración glomerular no se realizó de rutina en el momento en que los pacientes que ingresaron a la UCICOR. No hubo diferencias entre el tiempo de hospitalización y tampoco se dializó a ninguno de los pacientes evaluados.

En cuanto a la gráfica de supervivencia, puede observarse que las curvas se separan rápidamente desde el primer día y alcanzan su mayor variación a los 11 días donde la supervivencia de los pacientes sin SCR1 es del $89 \%$ y la de los pacientes con SCR1 es del $62 \%$. Luego de los 11 días y hasta los 30 días, la supervivencia se mantiene constante en ambos grupos, reafirmando el hecho de que los pacientes con SCR1 tienen una mayor mortalidad sobre todo en los primeros días de hospitalización ${ }^{33,34}$.

En cuanto a las limitaciones de este estudio, tenemos que hubo pacientes con diagnóstico de IC aguda que no fueron hospitalizados en la UCICOR, sino que pasaron a pabellones de medicina interna, ya sea porque no fueron considerados graves, ya sea por falta de disponibilidad de camas en esta unidad, y se desconoce si en este tipo de pacientes la evolución es más o menos favorable o si tienen una mayor o menor incidencia de SCR1 que en la UCICOR.

Otra limitación fue que, si bien se agregaron muchos criterios de exclusión para evitar que pacientes con ERC previa ingresaran al estudio, es posible que algunos pacientes hayan sido excluidos innecesariamente, como podría ser el caso de los pacientes considerados como ERC previa por el médico que realizó la historia clínica al ingreso del paciente a la UCICOR.

Los pacientes con hemoglobina $<10 \mathrm{mg} / \mathrm{dL}$ fueron excluidos de este estudio para evitar que pacientes con ERC no diagnosticada (que usualmente tienen hemoglobinas bajas) fueran incluidos en el mismo y también para evitar que la presencia de una baja hemoglobina sea otro factor confusor ya que al haber menos hemoglobina hay menor transporte de oxígeno y por lo tanto todos los tejidos incluidos el riñón sufren de una menor oxigenación. Sin embargo, este estudio mostró una asociación entre una mayor hemoglobina y la presencia de SCR1, que si bien no concuerda con la fisiopatología propuesta para el desarrollo de síndrome cardiorrenal en general, nos indica que en la fisiopatología del
SCR1 la anemia no es una variable asociada de forma inversa (a menor hemoglobina, mayor frecuencia de síndrome cardiorrenal) como sí lo es en el síndrome cardiorrenal crónico o tipo $2^{35}$. Esta asociación inversa ha sido ya observada en un estudio donde se demostró que la hemoconcentración durante la primera semana de los pacientes que ingresan con falla cardíaca agudamente descompensada se asoció a una peor función renal ${ }^{36}$. Otro estudio demostró también que niveles de hemoglobina más altos estuvieron asociados a disminuciones sutiles de la función renal en estadios tempranos de $\mathrm{ERC}^{37}$. Incluso en pacientes con ERC hay estudios que han demostrado que corregir la hemoglobina puede producir exacerbación de la falla cardíaca, mayor tasa de eventos cardiovasculares y muerte ${ }^{38}$, lo que nos hace dudar de que la relación directa entre los valores de hemoglobina y el síndrome cardiorrenal agudo esté totalmente definida.

Otras limitaciones de este estudio son su carácter observacional retrospectivo y el tamaño de la población.

En cuanto a la calidad del registro, la UCICOR del Hospital Nacional Arzobispo Loayza cuenta desde hace muchos años con un formato de historia clínica que permitió una recolección de datos homogénea para todos los pacientes. Sin embargo, lo ideal sería tener datos más detallados y si es posible guardados en formato digital para el futuro.

\section{Conclusiones}

La incidencia de SCR1 en la UCICOR encontrada en nuestro estudio es similar a la descrita en estudios extranjeros. La presencia de shock cardiogénico como causa de falla cardíaca y valores más altos de hemoglobina se asociaron a una mayor incidencia de SCR1. Aunque la asociación estadística entre el antecedente de ACV y la presencia de SCR1 no fue significativa al hacer el análisis multivariado, estudios previos demostraron que sí hubo asociación, por lo que en nuestro medio deberían realizarse estudios prospectivos con una población más numerosa para lograr confirmar esta relación. Los pacientes con SCR1 tuvieron mayor mortalidad intrahospitalaria a 30 días.

\section{Responsabilidades éticas}

Protección de personas y animales. Los autores declaran que para esta investigación no se han realizado experimentos en seres humanos ni en animales.

Confidencialidad de los datos. Los autores declaran que en este artículo no aparecen datos de pacientes.

Derecho a la privacidad y consentimiento informado. Los autores han obtenido el consentimiento informado de los pacientes y/o sujetos referidos en el artículo. Este documento obra en poder del autor de correspondencia.

\section{Financiación}

Ninguna. 


\section{Conflicto de intereses}

Los autores declaran no tener conflicto de intereses.

\section{Agradecimientos}

A la Dra. Elisa Juárez por su apoyo en las traducciones del español al inglés y a la Dra. Paola óliver por su apoyo en la formulación del proyecto.

\section{Bibliografía}

1. Pang PS, Komajda M, Gheorghiade $M$. The current and future management of acute heart failure syndromes. Eur Heart J. 2010;31:784-93.

2. Sliwa K, Davison BA, Mayosi BM, et al. Readmission and death after an acute heart failure event: predictors and outcomes in sub-Saharan Africa: Results fromthe THESUS-HF registry. Eur Heart J. 2013;34:3151-9.

3. MCMurray JJV, Adamopoulos S, Anker SD, et al. ESC guidelines for the diagnosis and treatment of acute and chronic heart failure 2012. Eur Heart J. 2012;33:1787-847.

4. Yancy CW, Jessup M, Bozkurt B, et al. ACCF/AHA guideline for the management of heart failure: Executive summary: A report of the American College of Cardiology Foundation/American Heart Association Task Force on Practice Guidelines. Circulation. 2013;128:1810-52.

5. Grupo de Estudios de Insuficiencia Cardiaca (GEIC) del Departamento de Clínica de la Sociedad Brasilera de Cardiologia y el Departamento de Directivas de la Sociedad Brasilera de Cardiologia. 1. ${ }^{a}$ Directiva Latinoamericana para la evaluación y conducta en la insuficiencia cardiaca descompensada. Insuf Card. 2006: 1.

6. Hunt SA, Abraham WT, Chin MH, et al. ACC/AHA 2005 guideline update for the diagnosis and management of chronic heart failure in the adult: A report of the American College of Cardiology/American Heart Association Task Force on Practice Guidelines (Writing Committee to Update the 2001 Guidelines for the Evaluation and Management of Heart Failure). Circulation. 2005;112:e154-235.

7. Dickstein K, Cohen-Solal A, Filippatos G, et al. Guía de prác tica clínica de la Sociedad Europea de Cardiología (ESC) para el diagnóstico y tratamiento de la insuficiencia cardiaca aguda y crónica (2008). Rev Esp Cardiol. 2008;61:1329.e1-70.

8. Ayón Seminario JE, Huayanay Falconí L, Postigo Díaz RM, et al. Insuficiencia cardiaca congestiva en el adulto mayor: experiencia clínica en el Hospital Nacional Cayetano Heredia, 1997-1999. Tesis: TB-UPCH WG370 A1.

9. Ronco C, Cicoira M, McCullough PA. Cardiorenal syndrome type 1: Pathophysiological crosstalk leading to combined heart and kidney dysfunction in the setting of acutely decompensated heart failure. J Am Coll Cardiol. 2012;60:1031-42.

10. House AA, Anand I, Bellomo R, et al. Definition and classification of cardio-renal syndromes: Workgroup statements from the 7th ADQI Consensus Conference. Nephrol Dial Transplant. 2010;25:1416-20.

11. McKee PA, Castelli WP, McNamara PM, et al. The natural history of congestive heart failure: The Framingham study. N Engl J Med. 1971;285:1441-6.

12. Jimeno Sainz A, Gil V, Merino J, et al. Validez de los criterios clínicos de Framingham para el diagnóstico de insuficiencia cardíaca sistólica. Rev Clin Esp. 2006;206:495-8.

13. Appleton C, Hatle L, Popp R. Relation of transmitral flow velocity patterns to left ventricular diastolic function: New insights from a combined hemodynamic and Doppler echocardiographic study. J Am Coll Cardiol. 1988;12:426-40.

14. Sohn D, Chai I, Lee D, et al. Assessment of mitral annulus velocity by doppler tissue imaging in the evaluation of left ventricular diastolic function. J Am Coll Cardiol. 1997;30:474-80.

15. Thomas J, Choong C, Flachskampf F, et al. Analysis of the early transmitral doppler velocity curve: Effect of primary physiologic changes and compensatory preload adjustment. J Am Coll Cardiol. 1990;16:644-55.

16. Myreng $Y$, Smiseth O, Risoe C. Left ventricular filling at elevated diastolic pressures: Relationship between transmitral doppler flow velocities and atrial contribution. Am Heart J. 1990;119:620-6.

17. Flachskampf F, Weyman A, Guerrero J, et al. Calculation of atrioventricular compliance from the mitral flow profile: Analytic and in vitro study. J Am Coll Cardiol. 1992;19:998-1004.

18. Little W, Ohno M, Kitzman D, et al. Determination of left ventricular chamber stiffness from the time for deceleration of early left ventricular filling. Circulation. 1995;92:1933-9.

19. Al-Omari MA, Finstuen J, Appleton CP, et al. Echocardiographic assessment of left ventricular diastolic function and filling pressure in atrial fibrillation. Echocardiographic assessment of left ventricular diastolic function and filling pressure in atrial fibrillation. Am J Cardiol. 2008;101:1759-65.

20. Dickstein K, Cohen-Solal A, Filippatos G, et al. ESC guidelines for the diagnosis and treatment of acute and chronic heart failure 2008. The Task Force for the Diagnosis and Treatment of Acute and Chronic Heart Failure 2008 of the European Society of Cardiology. Eur Heart J. 2008;29:2388-442.

21. Hunt SA, Abraham WT, Chin MH, et al. Focused update incorporated unto the ACC/AHA 2005 guidelines for the diagnosis and management of heart failure in adults: A report of the American College of Cardiology Foundation/American Heart Association Task Force on Practice Guidelines: Developed in collaboration with the International Society for Heart and Lung Transplantation. Circulation. 2009;119:e391-479.

22. Mehta RL, Kellum JA, Shah SV, et al., The Acute Kidney Injury Network. Acute Kidney Injury Network: Report of an initiative to improve outcomes in acute kidney injury. Crit Care. 2007:11:R31.

23. Alvelos M, Pimentel R, Pinho E, et al. Neutrophil gelatinaseassociated lipocalin in the diagnosis of type 1 cardio-renal syndrome in the general ward. Clin J Am Soc Nephrol. 2011;6:476-81

24. Bagshaw SM, George C, Dinu I, et al. A multi-centre evaluation of the RIFLE criteria for early acute kidney injury in critically ill patients. Nephrol Dial Transplant. 2008;23:1203-10.

25. Clec'h C, Gonzalez F, Lautrette A, et al. Multiple-center evaluation of mortality associated with acute kidney injury in critically ill patients: A competing risks analysis. Crit Care. 2011;15: R128.

26. Bagshaw SM, George C, Bellomo R, for the ANZICS Database Management Committee. Early acute kidney injury and sepsis: A multicentre evaluation. Crit Care. 2008;12:R47.

27. Bagshaw SM, Cruz DN, Aspromonte N, et al. Epidemiology of cardio-renal syndromes: Workgroup statements from the 7th ADQI Consensus Conference. Nephrol Dial Transplant. 2010;25:1406-16.

28. Forman DE, Butler J, Wang $\mathrm{Y}$, et al. Incidence, predictors at admission, and impact of worsening renal function among patients hospitalized with heart failure. J Am Coll Cardiol. 2004;43:61-7.

29. Du Y, Li X, Liu B. Advances in pathogenesis and current therapeutic strategies for cardiorenal syndrome. Life Sci. 2014;99:1-6 [consultado 20 Jul 2014]. Disponible en: http://dx.doi.org/ $10.1016 / j . l f s .2013 .10 .003$

30. Ronco C, Haapio M, House AA, et al. Cardiorenal syndrome. J Am Coll Cardiol. 2008;52:1527-39. 
31. Eshaghian S, Horwich TB, Fonarow GC. Relation of loop diuretic dose to mortality in advanced heart failure. Am J Cardiol. 2006;97:1759-64.

32. Echemann M, Zannad F, Briancon S, et al. Determinants of angiotensin-converting enzyme inhibitor prescription in severe heart failure with left ventricular systolic dysfunction: The EPICAL study. Am Heart J. 2000;139:624-63.

33. Damman K, Navis G, Voors AA, et al. Worsening renal function and prognosis in heart failure: Systematic review and metaanalysis. J Card Fail. 2007;13:599-608.

34. Bruetto RG, Rodrigues FB, Torres Ulysses $S$, et al. Renal function at hospital admission and mortality due to acute kidney injury after myocardial infarction. 2012. DOI: 10.1371/journal.pone.0035496 [consultado 30 Jul 2014]. Disponible en: http://www. plosone.org/article/info\%3Adoi\%2F10.1371\% 2Fjournal.pone.0035496
35. Libório AB, Uchia RS, Aragão AP, et al. Importance of anemia in the chronic cardiorenal syndrome: Effects on renal function after heart transplantation. Med Sci Monit. 2012;18: CR611-6.

36. Van der Meer P, Postmus D, Ponikowski P, et al. The predictive value of short-term changes in hemoglobin concentration in patients presenting with acute decompensated heart failure. J Am Coll Cardiol. 2013;61:1973-81.

37. Oh SW, Baek SH, Kim YC, et al. Higher hemoglobin level is associated with subtle declines in renal function and presence of cardiorenal risk factors in early CKD stages. Nephrol Dial Transplant. 2012;27:267-75.

38. Singh AK, Szczech L, Tang KL, et al., CHOIR Investigators. Correction of anemia with epoetin alfa in chronic kidney disease. N Engl J Med. 2006;355:2085-98. 